\title{
La formation pratique des enseignants au Cameroun
}

Marcelline Djeumeni Tchamabe

Université Laval

\section{Chronique internationale}

\section{Introduction}

Le Cameroun est un État unitaire, démocratique et bilingue. Il compte deux sous-systèmes (anglophone et francophone hérités de la colonisation française et anglaise) et deux langues officielles qui sont aussi des langues de scolarisation. Le système de formation est centralisé et déconcentré. Il apparait clairement que la décision de former préoccupe les décideurs, qu'il s'agisse de formation théorique ou pratique. Pour ce qui concerne particulièrement la formation pratique des enseignants qui nous préoccupe dans cette étude, des stratégies de formation sont définies et mises en œuvre à travers des documents tels que la Stratégie nationale de l'éducation (2010-2015; 2016-2020). La formation pratique professionnelle initiale des enseignants est considérée comme un enjeu primordial face à la demande croissante de la qualité en éducation, des exigences de l'innovation et les impératifs de la coopération et de la formation par alternance. Nous articulons ce travail autour du système de formation pratique des enseignants au Cameroun. Nous analyserons ses forces et ses faiblesses pour en dégager des perspectives après avoir présenté brièvement le système national de formation des enseignants des niveaux de la maternelle, du primaire et du secondaire. 


\section{Présentation du système de professionnalisation pratique des enseignants.}

Dans le cadre de la formation professionnelle, comme celle des enseignants, la professionnalisation regroupe l'ensemble des démarches et des actions qui permettent de maîtriser une activité et de la faire reconnaître comme une qualification et un métier. C'est aussi l'acquisition et la mise en œuvre de ces savoir-faire par une personne. De manière générale, la professionnalisation s'articule autour de trois grands processus simultanés : la professionnalisation des activités et des services qui permet le passage d'activités correspondant à des besoins nouveaux ou non satisfaits à des emplois repérables dans un système de classification des emplois et des compétences; la professionnalisation des salariés qui leur permet d'acquérir et de développer des compétences nécessaires à l'exercice de leurs activités, reconnues et transférables pour permettre leur maintien dans l'emploi ou une mobilité professionnelle; la professionnalisation des structures qui permet à la structure de formaliser son projet, de nouveaux services, de prévoir l'organisation de travail qui y répond et de suivre ses caractéristiques et son évolution.

Le concept de professionnalisation renvoie aux dimensions sociologiques, individuelles et pédagogiques (Ardouin, 2003, p. 3-4). Dans le sens sociologique, la professionnalisation vise à donner aux acteurs concernés un niveau de formation, de connaissances et d'éthique élevé pour faire d'eux de véritables professionnels regroupés dans un corps de métier, et reconnus par la société en général. Dans la dimension individuelle, la professionnalisation vise à accroître les savoirs, les savoir-faire des acteurs concernés. Dans le sens pédagogique, il s'agit de renforcer la dimension opératoire de la formation par des activités et contenus professionnels. Au demeurant, une formation professionnalisante accompagne la production de compétences professionnelles en situation. C'est un processus d'accompagnement et de construction d'identité professionnelle.

La formation pratique consiste donc dans le contexte réel de travail empirique et de terrain de développement d'une identité professionnelle individuelle et sociale avec l'intégration d'une réalité professionnelle vivante, concrète et réelle. Ainsi, face à la formation pratique,l'enseignant, futuréducateur, apprend les ficelles du métier. Il planifie, anticipe, gère la classe et la pédagogie. Pour Le Boterf (2002), les stages pratiques permettent à l'enseignant de mobiliser adéquatement les ressources acquises en formation théorique pour les intégrer dans les différents aspects de sa pratique professionnelle. Et on relève un impact des programmes de formation en stage sur la qualité des pratiques professionnelles des enseignants lors des stages ainsi que de la qualité de l'accompagnement des stagiaires en formation initiale selon Goupil et Lusignan (1993).

\subsection{Les programmes de formation pratique des enseignants}

En formation pratique, l'apprentissage est, pour une large part, une activité de traitement de l'information dans laquelle l'information concernant la structure du comportement et les évènements de l'environnement est transformée en représentations symboliques qui servent de guide pour l'action. Pour que le stagiaire intègre ces processus, il est accompagné par des professionnels plus expérimentés qui, dans le cas des formations par alternance, garantissent la transmission de la tradition d'un métier. Dans le cas du Cameroun, il s'agit d'un travail d'équipe entre maitres de stage, encadreurs des écoles annexes de formation ou des lycées et des collèges et superviseurs des services centraux de l'éducation. 


\subsection{L'accompagnement des stagiaires}

Le stage prend la forme de l'alternance théorie-pratique impliquant les aspects de l'accompagnement que sont le « coaching ", le mentorat, le tutorat ou la supervision. La relation de coaching professionnel a un rapport avec la qualité des pratiques professionnelles des enseignants en stage. Cette relation laisse présager l'importance des réseaux de soutien ainsi que des communautés d'apprentissage du métier pour que la professionnalisation se déploie aisément. Le mentor est également appelé tuteur par certains auteurs: Dumoulin $(2004$, p. 22) le définit comme un «soutien professionnel [...] de la part d'un collègue expérimenté (mentor)». Les chercheurs soulignent le fait que le mentorat constitue un moyen particulièrement efficace pour accompagner et soutenir l'enseignant en processus de professionnalisation (Fonkoua, 2005). Martineau et Vallerand (2005) définissent la période de formation pratique comme « une expérience de vie au travail qui implique un processus d'adaptation et d'évolution » durant la période de stage professionnel. L'apprenti-enseignant se familiarise avec son nouveau milieu professionnel, ses habitudes, son fonctionnement, ses rouages, sa culture et tout ce qui est en lien avec sa profession en tant que professionnel (Baillauquès et Breuse, 1993; Treleven, 2000). Par cet accompagnement, la formation pratique s'appréhende comme un apprentissage par observation de modèle ou modelage. L'imitation du modèle se fait à travers plusieurs types de stages : les stages d'observation, de tutelle et les stages en responsabilité.

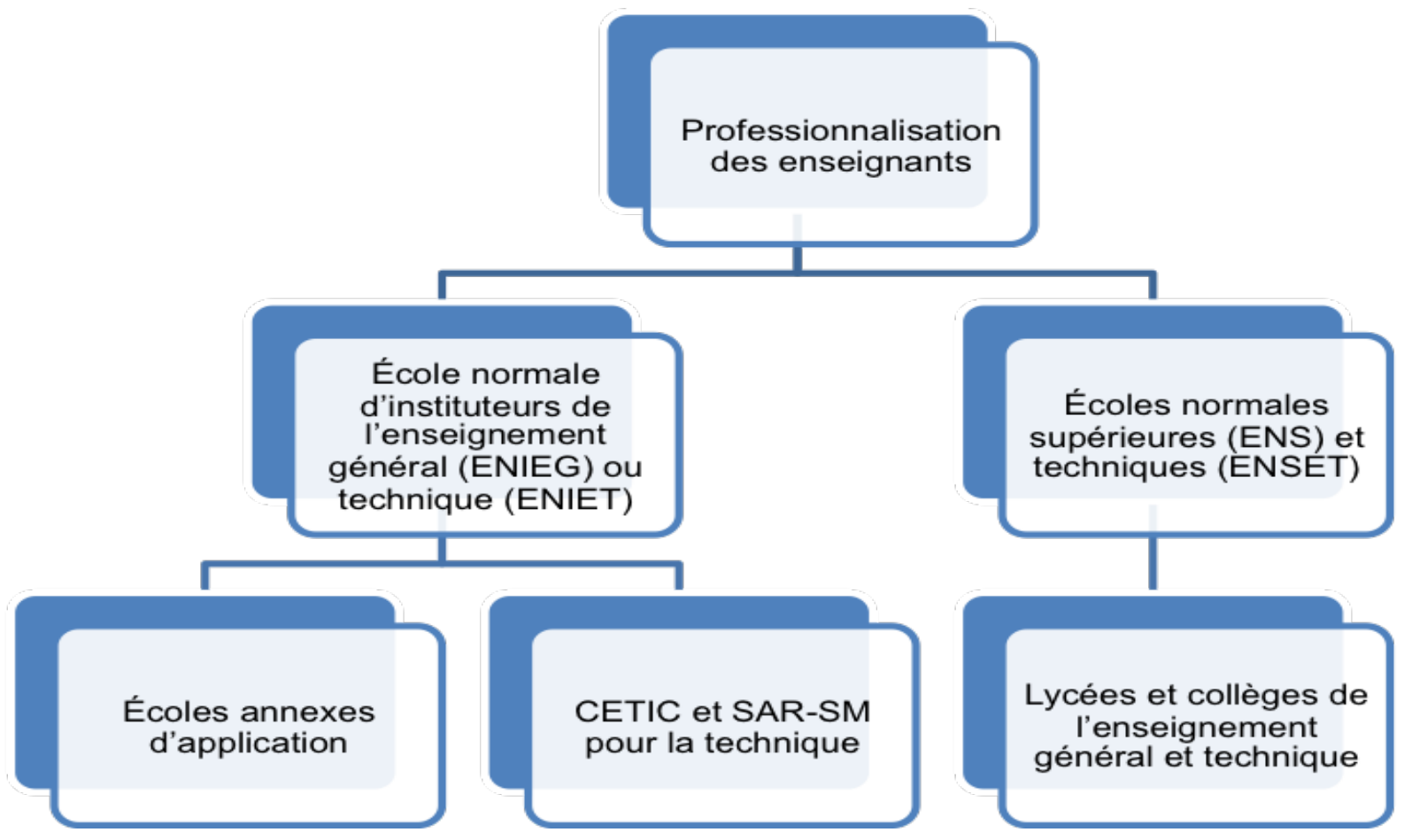

Figure 1

Système de formation pratique des enseignants au Cameroun. 
La formation pratique se déroule dans plusieurs types d'institutions : les écoles annexes d'application, les collèges d'enseignement technique industriel et commercial (CETIC), les sections artisanale et rurale et la section ménagère (SAR-SM) pour ce qui concerne les enseignants de la maternelle, du primaire et de l'enseignement technique; pour les autres enseignants (les élèves-professeurs), les stages ont lieu dans les lycées et les collèges. Ces trois stages (d'observation, en tutelle et stages en responsabilité) se déroulent suivant un système de progression linéaire que nous présentons ci-dessous :

\begin{tabular}{|c|c|c|c|c|c|c|c|c|c|c|c|}
\hline \multicolumn{12}{|c|}{ Formation pratique des maitres ou instituteurs et des professeurs } \\
\hline \multicolumn{3}{|c|}{$\begin{array}{l}\text { ENIEG ou ENIET } 1 \\
\text { Stage d'observation }\end{array}$} & \multicolumn{4}{|c|}{$\begin{array}{l}\text { ENIEG } 2 \text { ou ENIET } 2 \\
\text { Stage en tutelle }\end{array}$} & \multicolumn{4}{|c|}{$\begin{array}{l}\text { ENIEG ou ENIET } 3 \\
\text { Stage en responsabilité }\end{array}$} & \multirow{2}{*}{$\begin{array}{l}\text { ENS - EN- } \\
\text { SET } \\
\text { ENS-1 }\end{array}$} \\
\hline $1-1$ & $1-2$ & $1-3$ & $2-1$ & $2-2$ & $2-3$ & $2-4$ & $3-1$ & $3-2$ & $3-3$ & $3-4$ & \\
\hline \multicolumn{3}{|c|}{ ENIEG 1-4 } & \multicolumn{4}{|c|}{ ENIEG 2-5 } & \multicolumn{4}{|c|}{ ENIEG 3-4 } & ENS-2 \\
\hline
\end{tabular}

Figure 2

Processus de déroulement de la formation pratique.

D'après cette figure, le stage se déroule en trois étapes. Chaque étape est constituée d'un dispositif élaboré pour atteindre certains objectifs de formation.

\section{- Le stage d'observation}

ENIEG 1-1: Il s'agit de l'étape de recrutement dans la profession. Il y a alors trois niveaux de recrutement dans la fonction par concours (BEPC 1, Probatoire 1 et Baccalauréat). Ces trois profils entrent ensemble (secondaire 5, 6 et 7). Ils sont chacun dans leur cohorte en ce qui concerne les cours théoriques.

ENIEG 1-2 : L'élément 2 concerne la formation et l'encadrement des futurs instituteurs en stage d'observation. Il se caractérise par la durée variable, les contenus existants, les objectifs précisés, les acteurs définis et le processus de formation clarifié (carrefour pédagogique).

ENIEG 1-3 : L'évaluation se fait selon les objectifs définis. Le stagiaire produit un rapport de fin de stage basé sur l'observation de certaines leçons en particulier. C'est ce rapport qui sera noté non pas par le maitre de stage, mais par le professeur de l'ENIEG (École normale d'instituteurs de l'enseignement général) ou ENIET (École normale d'instituteurs de l'enseignement technique).

ENIEG 1-4 : Les forces de ce stage sont que les stagiaires sont présents dans les classes. Ils participent aux rencontres et aux carrefours pédagogiques. Ils observent leurs collègues qui sont en stage de responsabilité ou de tutelle, car les groupes sont mixtes. Les enjeux des stages pratiques d'observation des futurs maitres concernent les effectifs des stagiaires pour un accompagnement individualisé et le fait qu'ils arrivent sans être outillés pour une observation participante, ont déclaré certains stagiaires à la suite de leur formation.

\section{- Stage de tutelle}

ENIEG 2-1: Les critères de participation au stage de tutelle des élèves-maitres sont les mêmes : avoir complété un stage d'observation. Mais parfois, cette étape intermédiaire entre l'observation et la responsabilité est escamotée selon les profils des stagiaires (niveau BEPC 2 et Probatoire 2 et 
Baccalauréat). Le profil 3 (bac) sera le groupe qui aura souvent tendance à sauter cette phase en regard de la durée de leur formation qui varie de 6 à 10 mois.

ENIEG 2-2 : La formation est de deux semaines pour tous, les contenus sont définis par le service de stage et de la scolarité; plusieurs acteurs sont impliqués du niveau central au niveau déconcentré.

ENIEG 2-3 : L'évaluation et l'attestation des acquis consistent aussi en un rapport de stage rédigé à la fin de chaque stage et remis aux enseignants de l'ENIEG.

ENIEG 2-4 : L'encadrement de la formation est confié aux maitres des écoles d'application; les journées pédagogiques, les séminaires, les leçons modèles sont utilisés par les encadreurs comme stratégies de formation.

ENIEG 2-5 : Une des forces de ce dispositif intermédiaire est que le stagiaire apprend progressivement à faire des préparations et des planifications de la classe. Il bénéficie à cet effet de la collaboration de ses pairs, des encadreurs de l'établissement (le chef d'établissement, les animateurs de niveaux et les maitres) ainsi que des autres encadreurs (professeurs de l'ENIEG, inspecteurs d'arrondissement et conseillers pédagogiques à divers niveaux). Un des enjeux identifiés du stage reste le nombre élevé d'élèves-maitres et la diversité des profils. Une des solutions envisagées consiste à se faire coacher par les pairs.

\section{- $\quad$ Stage pratique en responsabilité}

ENIEG 3-1: Les critères de participation au stage de responsabilité par les élèves-maitres consistent en la formation pour la finalisation du parcours des instituteurs ayant passé au moins les deux phases de stage et rempli toutes les conditions d'admission en classe supérieure.

ENIEG 3-2 : Le contenu de la formation est défini par une note de mise en stage. Compte tenu de sa place pour l'examen final, les contenus sont renforcés et portent sur la préparation et la présentation des leçons qui seront évaluées pour attribution d'une note finale de formation pratique.

ENIEG 3-3 : L'évaluation, l'attestation des acquis et la certification de la formation pratique sont faites par une équipe nommée par le ministère formée des maitres de stage, des professeurs des ENIEG/ ENIET et des superviseurs. Des grilles d'évaluation sont fournies et un stagiaire qui ne satisfait pas aux exigences sera appelé à reprendre son stage pratique. La note de stage constitue $30 \%$ de la formation totale.

ENIEG 3-4: Lencadrement est assuré par le maitre de stage dans la pratique quotidienne de la classe. Le stagiaire prend en main la classe dans tous ses aspects de gestion des apprentissages et de la discipline. Il planifie, donne, évalue et anime toutes les activités de la classe.

ENIEG 3-5 : Les forces de ce stage en responsabilité des élèves-maitres est que l'évaluation est menée par un collège d'évaluateurs pour diminuer la subjectivité et assurer la validité des épreuves passées. L'évaluation finale constitue $30 \%$ de l'examen de sortie. Un des enjeux ici concerne l'inégalité du dispositif. À l'évaluation, les élèves-maitres de profil 1 ont effectué environ six stages alors que le profil 2 aura à son actif quatre stages environ et les autres un ou deux stages. La formation pratique des élèves-maitres de profil 3 (Bac) est jugée insuffisante pour adopter une professionnalité pertinente. 


\section{CHROANIOLIE}

\section{Les stages pratiques des élèves-professeurs des ENS et ENSET ou Écoles normales supérieures (enseignements généraux et techniques)}

Ces deux institutions forment les enseignants pour le niveau secondaire de l'enseignement général et technique. Deux cycles de formation sont offerts dans ces institutions. Le cycle 1 recrute les enseignants pour l'enseignement dans le premier cycle de l'enseignement secondaire. La formation se fait en six semestres : il s'agit d'une formation professionnelle et académique pour l'obtention conjointe du diplôme professionnel et d'une licence dans une discipline ou filière de spécialisation.

Le second cycle accueille divers profils : l'offre de formation concerne le recrutement direct dans le second cycle après la fin de formation au cycle 1 ; le retour sur titre des enseignants venant du terrain et le recrutement par concours des titulaires d'une licence dans une discipline de spécialisation dans la formation des élèves. La formation pratique des élèves-professeurs se déroule selon les phases d'imprégnation et de consolidation pour les deux groupes.

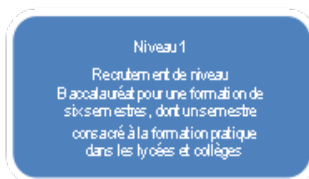

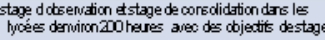

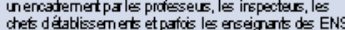
edes ENSET.

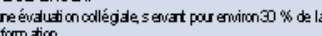

armation

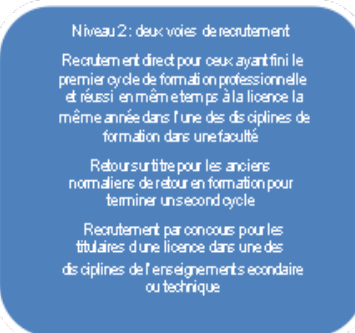

stage doks evadion estage decoredidation

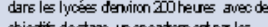
cojectio destage, unencaremertpa détalissemert et patois les eresigats des ENS d de ENSET.

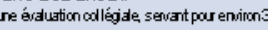
W delatomition

unporil deresigate dues

pe destage pourles retar surtithe

rédactiondeniqpoct des taze érádúparle

ire pedteus auriveascerta

une fiche deradution aree des cittives

dévalution

Figure 3

Stage pratique des élèves-professeurs.

\section{Les défis de la formation pratique des enseignants}

Les défis identifiés sont nombreux. Nous en analyserons quelques-uns en les subdivisant en trois points : les défis liés au recrutement, à l'encadrement des stages et à l'évaluation de la formation pratique des enseignants camerounais. 


\subsection{Les défis liés au recrutement des enseignants à former}

\section{- Le recrutement des élèves-maitres et des élèves-professeurs}

L'accès à ces écoles de formation se fait par concours, mais la situation des élèves-professeurs est quelque peu différente de celle des élèves-maitres. Le concours des premiers donne accès directement à la fonction publique et garantit un emploi. Cette formation est encore l'un des rares à déboucher sur la fonction publique camerounaise et une intégration ipso facto dans l'administration avec une garantie de salaire et une retraite à l'âge de 60 ans. Ces conditions font du métier d'enseignant une fonction très attrayante dans un contexte dominé par le chômage des jeunes diplômés et le déclassement de plusieurs professions (Fonkoua, 2005). Les conséquences de cette situation sont que cette attractivité peut avoir un effet inverse sur la profession, car elle attire tous les demandeurs d'emploi et une baisse de la compétitivité. Les concours attirent chaque année des milliers de candidats, mais les formateurs comme les utilisateurs des produits remettent en cause les niveaux des élèves-professeurs, ce qui a un impact sur la qualité de la formation aussi bien théorique que pratique et sur l'insertion professionnelle. Un défi important concerne la sélection des enseignants qui sont recrutés pour la formation pratique. On constate un niveau d'entrée trop faible, des profils trop disparates et des intentions professionnelles non clarifiées que certains candidats à l'enseignement utilisent pour accéder à la fonction publique et aux autres métiers qui ne leur semblent pas accessibles. Un enseignant, comme tous les fonctionnaires de l'État, peut, grâce à la permissivité de la fonction publique, servir dans tous les autres corps de métiers de son portefeuille.

Contrairement à l'enseignement secondaire, l'enseignement primaire ne recrute plus directement des fonctionnaires. Les élèves-maitres doivent attendre le lancement éventuel d'un recrutement pour être contractualisé. Depuis 1995, la crise économique a entrainé le gel de recrutement par l'État du Cameroun. La coopération bilatérale et les bailleurs de fonds comme la Banque mondiale ont ainsi financé l'admission dans la profession de plusieurs enseignants formés. Dans ce niveau, le défi est celui des catégories diverses et variées des personnels. Même s'ils reçoivent tous la même formation, les maitres ont des prestations salariales différentes selon que l'on est contractuel, contractualisé ou fonctionnaire. Dernièrement, certains acteurs ont décrié l'engagement dans la formation aussi bien théorique que pratique des futurs éducateurs en raison de cette situation. Un défi d'harmonisation des statuts dans ce secteur s'impose pour améliorer la qualité de l'éducation de base.

\subsection{L'encadrement de la formation pratique}

\section{- L'encadrement des élèves-maitres}

Le nombre d'encadreurs est nettement insuffisant. Ils ont également des statuts différents comme nous l'avons démontré plus haut : instituteurs permanents, fonctionnaires, contractuels et contractualisés, vacataires ou maitres des parents. En plus des défis que nous avons déjà relevés, nous avons ce déficit d'encadrement pratique des enseignants. De Jomtien en 1990 et Dakar en 1994, les pays africains se sont approprié des idées de l'UNESCO et se sont engagés à l'initiative de l'Éducation pour tous (EPT) à l'horizon 2015. Une conséquence de cette politique éducative adoptée par le Cameroun est l'ouverture des écoles de formation dans tous les chefs-lieux de département pour une meilleure 
couverture de la carte scolaire des régions du pays. Le Cameroun est passé à 58 écoles de formations des instituteurs de l'enseignement général et à 2 écoles de l'enseignement technique.

Cependant, l'enseignement dans les ENIEG/ENIET est un enseignement post-BEPC, c'est-à-dire correspondant au second cycle de l'enseignement secondaire pour la grande majorité des encadreurs. C'est pourquoi le niveau de qualification des formateurs pratiques des élèves-maitres au Cameroun peut comporter certaines limites. L'on se fonde sur l'expérience de l'enseignement pour leur confier des stagiaires. Cependant, leur niveau de qualification dans les disciplines en ce qui concerne la maitrise des contenus disciplinaires peut être mis en doute. En même temps, la formation initiale ou continue des enseignants fait face à des enjeux et à des défis majeurs. De nos jours, les enseignants camerounais doivent relever sur le plan professionnel des défis d'une éducation de qualité. Les États généraux de l'éducation, les projets Éducation pour tous (EPT) et les Objectifs du Millénaire pour le développement concourent à mettre en exergue le fait qu'en Afrique subsaharienne et au Cameroun, l'éducation souffre d'un déficit en quantité et en qualité d'enseignants formés. Plus de quatre millions d'enfants en Afrique subsaharienne n'entreront pas dans une salle de classe par manque d'enseignants. Sur un plan plus qualitatif, des élèves quittent l'école primaire sans savoir ni lire, ni écrire, ni calculer, qui sont pourtant des compétences de base nécessaires pour une poursuite de la scolarité de base. En effet, les réflexions telles que celles menées lors des États généraux de l'éducation (1995) s'accordent de manière unanime sur le fait que l'école camerounaise a un faible rendement interne et externe (Banque mondiale). En ce qui concerne le rendement interne, il en ressort selon la même source que les taux de déperdition sont élevés et les redoublements avoisinent les $40 \%$. De plus, selon Educ'Avenir (2007), le rendement externe montre que le système éducatif n'est pas opérationnel : les produits du système éducatif s'insèrent difficilement dans le tissu économique. Une meilleure formation pratique des enseignants doit permettre d'améliorer la qualité de l'éducation et le développement du Cameroun (Banque mondiale, 2014). Ces hypothèses ont entrainé plusieurs réformes dans les systèmes de formation des enseignants, notamment la mise sur pied d'équipe d'encadrement de stages pratiques formés des maitres d'application, des professeurs des ENIEG et des superviseurs (inspections et délégations à divers niveaux). Un défi de la formation pratique des enseignants serait une réforme du fonctionnement pédagogique de ces équipes dans le sens d'une meilleure définition des finalités de chaque type de stage (observation, tutelle, ou responsabilité), des contenus, de la durée, des modalités et des processus de la formation et de l'évaluation des enseignants au Cameroun pour relever les grands défis d'universalisation en ce qui concerne l'éducation de base de ce siècle. Généralement, la durée des trois stages chez les instituteurs de niveau BAC n'atteint pas les quotas horaires prévus.

\section{- L'encadrement des élèves-professeurs}

Plusieurs nouvelles institutions de formations ont vu le jour au Cameroun pour la formation des élèves-professeurs. Ces institutions de formations des enseignants au Cameroun sont passées à $\mathrm{n}=5$ en 2014. Contrairement aux élèves-maitres, les élèves-professeurs ne sont plus formés dans les lycées d'application. Leur dispositif de formation comprend pour l'encadrement le professeur chargé de la formation pratique à qui est confié le stagiaire, mais aussi un professeur de l'ENS et un superviseur (inspecteur national). Ces deux derniers n'interviennent généralement qu'à la fin pour évaluer la formation pratique. On remarque néanmoins dans ce dispositif quelques failles concernant notamment les nouvelles disciplines nées de la réforme de l'éducation et pour lesquelles on n'a pas encore déterminé de compétences sur le terrain. 
En effet, une loi de l'orientation est adoptée en 1998 qui renforce le rôle de l'orientation scolaire, universitaire et professionnelle pour la formation de qualité des Camerounais enracinés dans leurs cultures, mais ouverts au monde. Cet engagement de l'État se concrétisera par des réflexions sur le système éducatif camerounais manifesté au travers des États généraux de l'éducation de 1995 par l'enrichissement du programme de formation avec deux nouveaux cours, Langue et culture camerounaise (LCC) et L'informatique dans l'enseignement secondaire (Djeumeni Tchamabé,2013). Pour ces disciplines, il y a d'énormes difficultés à trouver des encadreurs pour les élèves-professeurs.

De même que pour ces innovations, des défis de la mondialisation, au cours de ces deux dernières décennies, commandent une révision curriculaire de la formation pratique des enseignants. Lélaboration de la stratégie du secteur de l'éducation en 2001 vient donner une nouvelle orientation à une politique de formation adéquate des enseignants à tous les niveaux du système éducatif. Elle apparaissait nécessaire dans la mesure où de nombreuses innovations pédagogiques ont été introduites lors des États généraux. En effet, la formation devait être améliorée dans le sens de l'intégration d'ateliers d'activités pratiques introduits dans les cursus de formation théoriques en vue d'allier théories et pratiques en formation initiale. La formation théorique est effectivement complétée par des stages pratiques dans les établissements dédiés. Ce dispositif de professionnalisation est malgré tout remis en question. Ces questionnements concernent des compétences effectivement acquises en formation pratique, le fonctionnement des dispositifs d'alternance, les évaluations des enseignants et les formations pratiques des enseignants qui sont admis directement dans les classes sans formation initiale et l'intégration des TIC pour la formation pratique initiale et continue des enseignants.

L'insuffisance de la formation pratique en général (200 heures) pour les élèves-professeurs et l'inégale couverture de ces heures sur l'ensemble des formés font que la formation pratique souffre de la grande variabilité des profils de recrutement des enseignants des Écoles normales supérieures et du profil académique de leurs formateurs. Les professeurs des ENS sont recrutés sur la base de leur doctorat dans leurs disciplines de spécialisation. La pédagogie universitaire n'entre pas dans les critères de recrutement. Face à cette réalité, l'on est confronté à la problématique de la transmission des connaissances et de leur exploitation opportune dans des contextes précis où les besoins changent constamment. Cette situation s'est peu à peu stabilisée et le curriculum prescrit et le curriculum réel (Perrenoud, 2001) tendent de plus en plus à s'harmoniser. Ceci a donné en 2015 une opportunité de réécrire un curriculum pour des ENS en l'actualisant et en l'aménageant en fonction de ces nouveaux développements qui doivent se traduire selon le processus de la transposition didactique.

\section{L'évaluation des formations pratiques des enseignants}

L'évaluation des apprentissages est fortement liée aux programmes notamment au niveau des programmes et des acquis scolaires des élèves-maitres et élèves-professeurs. Ceux-ci incitent à revoir le curriculum en prenant en considération ses points forts et les insuffisances constatées : parmi les constats négatifs récurrents dans l'ensemble des deux sous-systèmes éducatifs, on trouve, par exemple, la surcharge des programmes, la restitution des savoirs au moment de l'évaluation et l'incapacité d'un nombre important d'élèves-maitres ou professeurs à résoudre des problèmes professionnels, la prééminence des méthodes transmissives, le recours à des enseignements et des apprentissages jugés peu significatifs. 
Face à ces critiques, en 2014 un nouveau programme a été élaboré pour la formation théorique et pratique des élèves-maitres (Ministère des Enseignements secondaires [MINESEC], 2014). Il s'agit d'un programme décliné avec une logique de compétences pour remplacer l'ancien qui était utilisé depuis trois décennies. Des compétences transversales et professionnelles sont définies pour permettre de mettre en œuvre une pédagogie de la maitrise.

Toutefois, la mise en place d'une rénovation pédagogique impliquant l'adoption d'une approche pédagogique donnée ne peut être fiable et efficiente que si elle fait l'objet d'une «contextualisation » garantissant son ancrage dans le pays où elle est implantée; cette prise en compte du contexte signifie, au plan micro, l'adaptation du curriculum aux données précises de la classe et des apprenants; mais au plan collectif et macrostructurel, elle se conçoit à plusieurs dimensions : politiques, organisationnels et pédagogique. Or, le nouveau programme a été expérimenté pendant une année, mais déjà l'évaluation des élèves-maitres montre des défis à relever dans les différentes dimensions : une politique d'évaluation basée sur des critères professionnels (gestion pédagogique et gestion de la classe); des dispositifs d'évaluation efficaces, efficients et cohérents; une organisation de collectes de données basées sur des indicateurs clairs; une formation des acteurs sur les valeurs de l'évaluation; une élaboration des référentiels d'évaluation pour accompagner les programmes, au niveau pédagogique des guides pour aider les enseignants dans l'évaluation théorique et pratique des élèves-maitres.

Des études sur la formation professionnelle montrent que l'expérience et une grande exposition au contexte d'enseignement font la différence dans la manière d'embrasser plus tard, dans l'exercice, les problèmes éducatifs. L'évaluation des élèves-maitres telle qu'elle se pratique au cours des stages pratiques que l'institution organise pendant la formation initiale ne prend pas en compte cet aspect. Une seule leçon est observée et notée et tous les aspects de la profession ne sont pas pris en compte. En outre, ils prennent davantage de temps pour réaliser les diverses tâches reliées à l'enseignement, que ce soit la planification des leçons, la préparation des évaluations, la correction des travaux d'élèves ou la mise en place d'un système de gestion (Renard, 2003). De nombreux facteurs sont à l'origine de ces difficultés. Par exemple, le temps imparti aux stages pratiques d'enseignement est court, il peut être une potentielle source de ces difficultés. Un des facteurs décisifs est d'ordre économique et concerne la mise en mission des évaluateurs. L'un des défis pourrait consister à une organisation des ressources locales au niveau des établissements de formation autour des animateurs pédagogiques et des services de scolarité et des stages, ce qui permettrait de résoudre l'autre problème handicapant des élèves-maitres qui est celui de l'inadéquation entre les dispositifs de formation et les activités d'enseignement sur le terrain.

\section{Conclusion et perspectives}

La formation pratique des enseignants au Cameroun et dans la majorité des pays en voie de développement reste une question vive comme l'analyse l'a démontré.Dans ses dimensions sociologiques, individuelles et pédagogiques, on a pu relever des forces et des faiblesses. On y rencontre de meilleures programmations de formation, l'existence de politique de formation pratique des enseignants et des dispositifs élaborés quant à ce qui est nécessaire pour la formation des enseignants. Mais, il ressort aussi que ce dispositif dans son ensemble reste encore trop théorique, et ne s'appuie que très peu sur la réalité empirique de l'enseignement, moments charnières où les élèves-maitres et les élèves- 
professeurs apprennent à anticiper des difficultés que pose au quotidien la situation d'enseignement; des capacités d'accueil inégales en formation pratique qui sont loin de couvrir les besoins du pays en enseignants qualifiés à tous les niveaux. La situation d'inadéquation entre objectifs de formation et projets éducatifs liés aux préoccupations spécifiques au Cameroun; parfois une incohérence du dispositif de professionnalisation, la multiplicité des acteurs qui rendent inopérants les dispositifs mis en place pour professionnaliser sur le plan sociologique et individuel et des contradictions dans les finalités de la formation pratique entre logiques des objectifs et des compétences. Afin de mieux comprendre ce phénomène de professionnalisation des enseignants camerounais, de futures études pourraient s'intéresser aux thèmes suivants :

1. Lélaboration d'un observatoire de bonnes pratiques de formation pratique des enseignants en Afrique subsaharienne pour améliorer la qualité de l'éducation, notamment l'étude des modèles d'alternance et de partenariats à mettre en place pour la formation des enseignants;

2. L'influence d'un dispositif de formation, sous-tendu par une longue période d'exposition aux stages pratiques et soutenu par des groupes d'entraide sur l'insertion professionnelle des enseignants;

3. L'élaboration et l'expérimentation de portfolio d'évaluation des objectifs de formation pratique orientées vers les compétences nécessaires à l'exercice de la fonction enseignante;

4. L'intégration des TIC dans la formation pratique - les modèles théoriques, les méthodes et les référents adaptés à la situation camerounaise;

5. Le développement d'une identité professionnelle chez les maitres de stage et encadreurs de la formation pratique des enseignants au Cameroun.

\section{Références}

Ardouin, T. (2003). Ingénierie de la formation pour l'entreprise. Paris : Dunod.

Baillauquès, S. et Breuse, E. (1993). La première classe. Paris : ESF.

Djeumeni Tchamabé, M. (2013). Enseignement de l'informatique au Cameroun : la loi du plus riche. Revue EPI, (157). Repéré à http://www.epi.asso.fr/revue/articles/a1309h.htm

Dumoulin, M.-J. (2004). Construire son expérience en situations indéterminées : préoccupations méthodologiques. Formation et Profession, 10(2), 21-24. Repéré à http://www.crifpe.ca/download/verify/243

Fonkoua, P. (2005). Les dispositifs de formation des enseignants. Dans Les institutions de formation des enseignants en Afrique sub-saharienne pour un renforcement des capacités (p. 109-119). Repéré à http://unesdoc.unesco.org/images/0014/001406/140665m.pdf

Goupil, G. et Lusignan, G. (1993). Apprentissage et enseignement en milieu scolaire. Montréal, QC : Gaëtan Morin.

Martineau, S. et Vallerand, A.-C. (2005). L'insertion professionnelle des enseignants : un enjeu pour le milieu scolaire, un défi pour le monde de la recherche. Communication présentée dans le cadre des séminaires du Centre de recherche interuniversitaire sur la formation et la profession enseignante (CRIFPE), Jouvence. Repéré à

http://www.insertion.qc.ca/IMG/pdf/Martineau et Vallerand 08 decembre 2005.pdf

Ministère des Enseignements secondaires (MINESEC). (2014). Programme des ENIEG. 
Perrenoud, P. (2001). Articulation théorie-pratique et formation des praticiens réflexifs en alternance. Dans P. Lhez., D. Millet et B. Séguier (dir.), Alternance et complexité en formation : éducation, santé, travail social (p. 10-27). Paris : Seli Arslan.

République du Cameroun. (1995). États généraux de l'éducation.

Renard, L. (2003). Setting new teachers up for failure... or success. Educational Leadership, 60(8), 62-64. Repéré à http://jan.ucc.nau.edu/ coesyl-p/principle13-article1.pdf

Treleven, C. G. (2000). Best practices in new teacher induction and their implications for Franklin Pierce school district (Mémoire de maîtrise inédit). Pacific Lutheran University, Tacoma (Washington). 